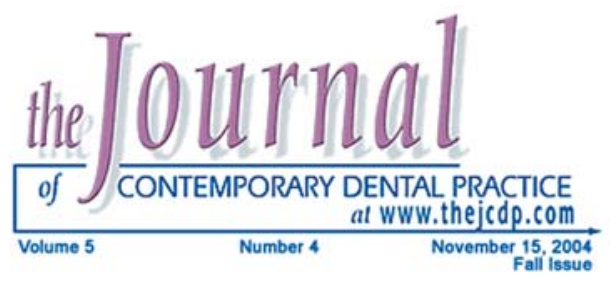

\title{
Labial Veneers in the Management of Desquamative Gingivitis: Report of a Case
}

\author{
Rampalli Viswa Chandra, BDS; Pratibha Pandurang, MDS: \\ Khandige Mahalinga Bhat, BSc, MDS
}

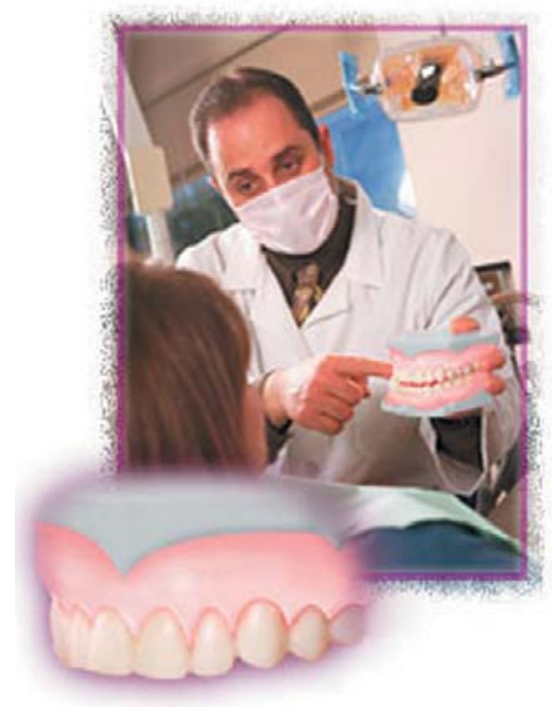

\section{Abstract}

Desquamative gingivitis is a condition characterized by intense erythema, ulceration, and desquamation of the free and attached gingiva. Approximately $50 \%$ of desquamative gingivitis cases occur on the gingival tissues though it is not uncommon at other intraoral and extraoral sites. Though topical corticosteroids are considered the mainstays in the treatment of desquamative gingivitis, the inability of these medicaments to be in contact with the affected sites for longer duration has been implicated as a factor in reducing the efficacy of this mode of treatment. The case presented here is of a 24-year old male with desquamative gingivitis of 3 years duration. Topical corticosteroid therapy using custom-made acrylic veneers was utilized in the patient with remarkable results. This paper highlights the use of acrylic labial veneers over the gingival tissue as a vehicle for delivering medication and as a way to improve aesthetics.

Keywords: Labial veneer, desquamative gingivitis, topical corticosteroids, erythema, mucous membrane pemphigoid, ulceration

Citation: Chandra RV, Pandurang P, Bhat KM. Labial Veneers in the Management of Desquamative Gingivitis: Report of a Case. J Contemp Dent Pract 2004 November;(5)4:122-132.

(c) Seer Publishing 


\section{Introduction}

The term Chronic Desquamative Gingivitis was coined by Prinz in 1932 to describe a condition characterized by intense erythema, desquamation, and ulceration of the free and the attached gingiva. ${ }^{1}$ Merritt described milder cases of this condition and further characterized the disease process. ${ }^{2}$ Approximately $50 \%$ of the desquamative gingivitis cases are localized in the gingiva though the involvement of the gingiva with other intraoral sites or extra oral sites is not uncommon. This condition is common in the anterior region and may be aggravated by plaque accumulation, trauma because of mastication, and by brushing of teeth. ${ }^{3}$

The extent and degree of gingival involvement varies in different cases. At times, there can be intense erythema, which predominates over desquamation. In other cases, desquamation is the main feature where the epithelium can be peeled away easily from non-ulcerated areas. Several cases show extensive areas of denudation with an exposed connective tissue surface. Patients may be asymptomatic or complain of a burning sensation with or without intense pain. The condition occurs more often in females than in males. ${ }^{3}$ While it can be seen at any age starting at puberty, most cases appear after 30 years of age. The disease is of indefinite duration with periods of remission and exacerbation. The gingiva may heal after several months or the condition may persist for years. ${ }^{4}$

Topical corticosteroids are considered one of the mainstays in the treatment of desquamative gingivitis. ${ }^{3,5}$ However, inability of the topical medicaments to be in contact with the affected sites for a longer duration has been implicated as a factor in reducing the efficacy of this mode of treatment. ${ }^{3}$ In order to maintain a constant contact of the topical corticosteroid with the affected tissue and also to improve the gingival aesthetics, the use of an acrylic labial veneer over the gingival tissue was assessed as this method is known to reduce most of the difficulties associated with topical drug therapy and may augment the response. ${ }^{5}$

\section{Case Report}

A 24-year old male agriculturist was referred to the dental clinic in April 2000 with a chief complaint of red swollen gums in the left maxillary region. The patient reported bleeding from the gums while eating food for the past 6 months. There was no associated pain. Upon examination, the lesions were tender. There was diffuse erythema of the gingiva in relation to the upper left canine-premolar region. The lesions were scrapable showing underlying erosions, which were sharply demarcated from the adjoining areas (Figure 1).

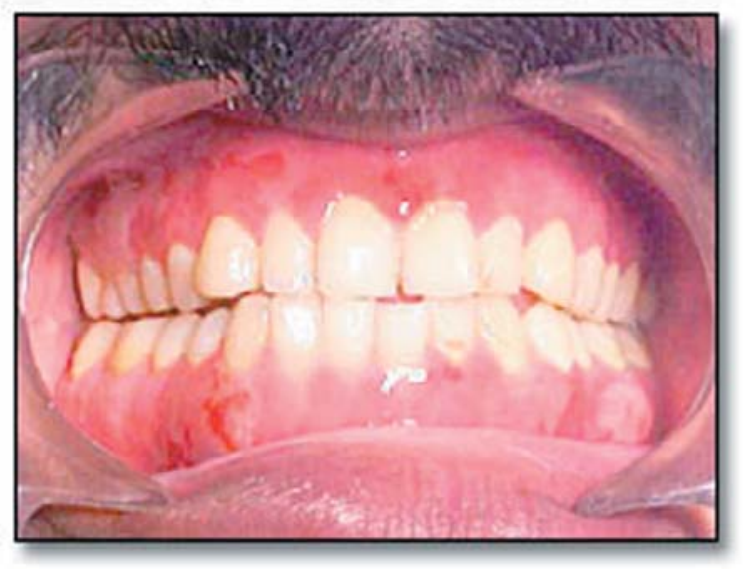

Figure 1. Initial Visit. There is erythema and desquamation in the upper and lower buccal areas. Nikolskys sign was positive.

There was associated glossitis. There were stains and calculus on the surface, but the lesions appeared unrelated to plaque accumulation. The patient also had Black's class II amalgam restorations on teeth number 16 and 46 . On questioning, the patient gave a history of pruritis of the skin with suppuration for a period of two years. There were itchy transient erythematous lesions on the trunk and the hands, which lasted for about half an hour. The patient was evaluated for the presence of genito-cutaneous lesions, presence of vesicles or bullae, gastrointestinal or respiratory problems, and for motor or sensory disturbances. However, no significant findings were present.

Lichen planus of the skin may result in pruritis. ${ }^{1}$ To obtain a definitive diagnosis, a direct immunoflouresence test was performed after obtaining a skin biopsy which was negative for IgG, IgA, IgM, $\mathrm{C}_{3}$, and fibrinogen. An allergen test was also carried out to rule out lichenoid reactions that are caused by contact allergy but it was negative.

Following this the patient did not return until 2002. In this interim period, he was subjected to innumerable forms of treatment including dapsone, corticosteroids, herbal preparations, and ayurvedic 


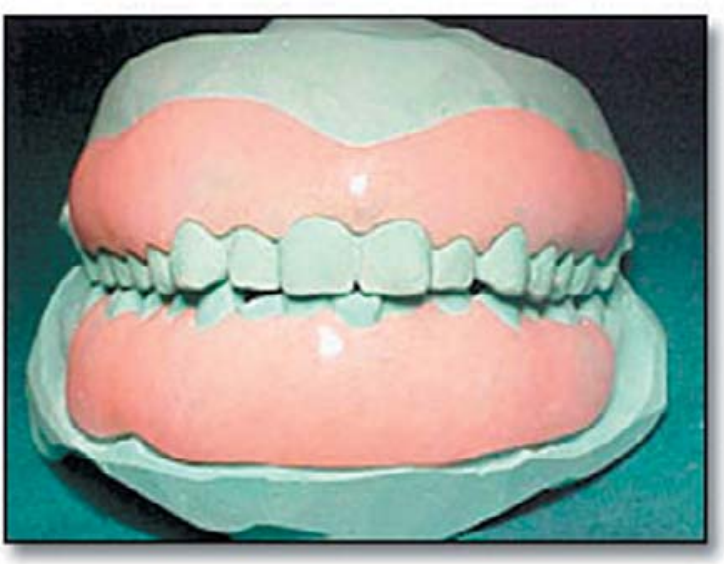

Figure 2. Veneers in both the maxillary and the mandibular arches extended from the mesiobuccal line angle of the second molar of one side to the mesiobuccal line angle of the second molar of the contralateral side.

treatment. His return followed several unsuccessful attempts to find a cure elsewhere. The patient did not have pruritis at this point of time. But he presented with increased erythema of the gingiva with four areas of desquamation, which were covered by a pseudomembrane. The patient also presented with a large superficial ulceration of the buccal mucosa near tooth number 46 caused by the rupture of a bulla. The lesions were also associated with spontaneous bleeding. Nikolskys sign was positive. At this time, the patient was provided with a diary to record his daily activities for a week to identify potential allergens, but his recordings were not significant.

A gingival biopsy was taken and was subjected to routine histopathological examination. The epithelium showed desquamation and subepithelial separation with areas of necrosis superior to the basement membrane. The basement membrane was intact, thus, ruling out the possibility of lichen planus. The gingival biopsy was also subjected to a direct immunoflouresence test, but the results were negative. Mucous membrane pemphigoid (MMP) was considered because oral lesions occur in over $90 \%$ of the patients with MMP. ${ }^{1,6}$ Desquamative gingivitis is the most common and may sometimes be the only manifestation of the disease. There may also be the presence of one or more intraoral vesicles as present in this patient, which was suggestive of MMP, though ulcerations of oral mucosa are more common. ${ }^{1,4,6}$ Oral pemphigoid is a subset of MMP characterized by low frequency of positive findings in immuno-

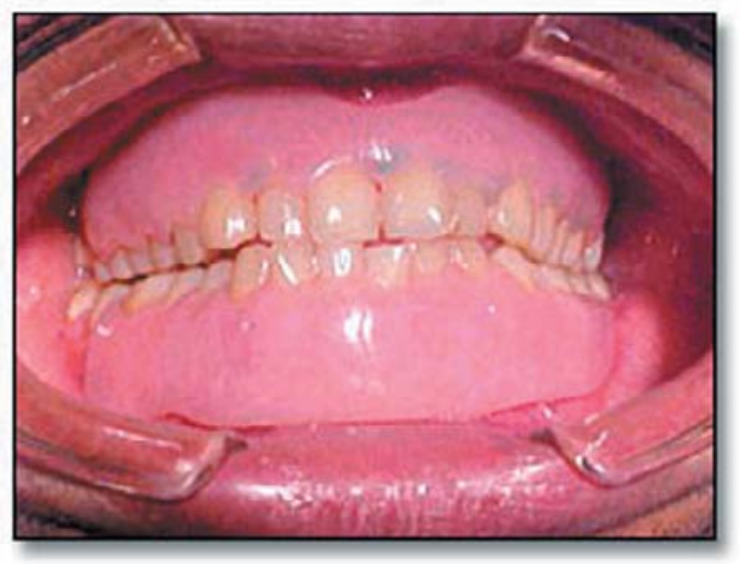

Figure 3. Veneers in both the maxillary and the mandibular arches extended from the mesiobuccal line angle of the second molar of one side to the mesiobuccal line angle of the second molar of the contralateral side.

flouresence and other tests and the presence of oral lesions only. ${ }^{6}$ Thus, a working diagnosis of desquamative gingivitis secondary to oral pemphigoid was made.

A thorough scaling was done and the patient was prescribed $0.2 \%$ chlorhexidine gluconate (Chlohex ${ }^{\circledast}$, Dr.Reddys labs) b.i.d. Oral hygiene instructions were also reinforced.

It was then decided topical corticosteroid therapy using custom made acrylic veneers be instituted in the patient. After thorough scaling, an alginate hydrocolloid impression of both the arches was obtained and the cast was poured in dental stone. Acrylic veneers were constructed using heat cured pink acrylic. The thickness was maintained at about $1 \mathrm{~mm}$ to obtain flexibility for the patient to insert and remove the appliance with a minimum of difficulty and extended from the free gingival margin to the depth of the vestibule. The veneers in both the maxillary and the mandibular casts extended from the mesiobuccal line angle of the second molar of one side to the mesiobuccal line angle of the second molar of the contralateral side (Figures 2 and 3).

The areas of desquamation determined the extent of this veneer. Undercuts distal to the canine were utilized, thus, making the appliance a selfretaining one. The tissue side was not polished but every care was taken to ensure there were no spicules or rough surfaces, which may injure the already fragile gingiva. The patient was 


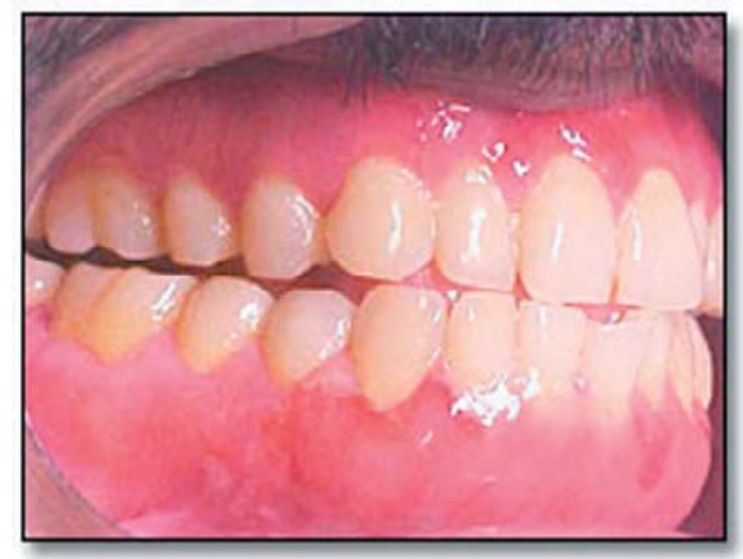

Figure 4. After 3 weeks. No apparent change in the condition apart from the reduction in the erythema.

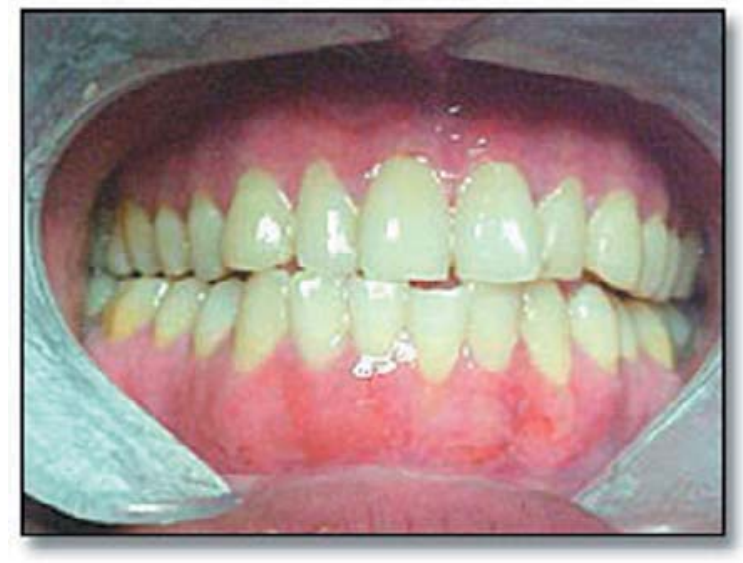

Figure 6. After 10 weeks. There is complete reduction in desquamation in all the sites except in the left canine region.

told to apply Clobetasol propionate $0.05 \%$ gel (Clobetamil ${ }^{\circledR}$, Merck) to the fitting surface and to wear the appliance q.i.d. each time retaining it for $1 / 2$ hour for an initial period of 4-6 weeks. He was asked to retain the saliva as long as possible and then to expectorate it. He was recalled after 3 weeks with no apparent change in the condition apart from the reduction in the erythema (Figure 4). The patient then returned after 7 weeks, and a reduction of desquamation was observed in the maxillary anterior and the mandibular left and right buccal sites (Figure 5).

By the tenth week, there was complete reduction in desquamation in all the sites except in the left canine region (Figures 6 and 7). The patient was regularly evaluated for the presence of candidiasis and oral prophylaxis was performed at every visit. The patient is using the appliance and is under evaluation.

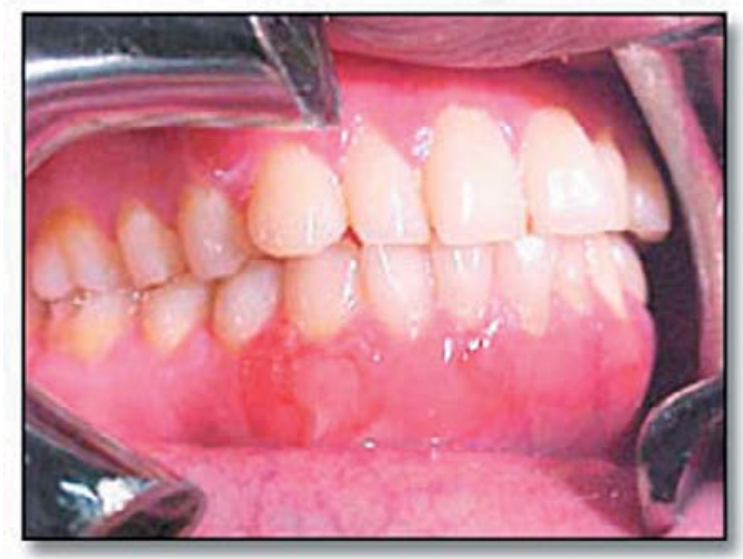

Figure 5. After 7 weeks. Reduction of desquamation in the maxillary anterior and the mandibular left and right buccal sites.

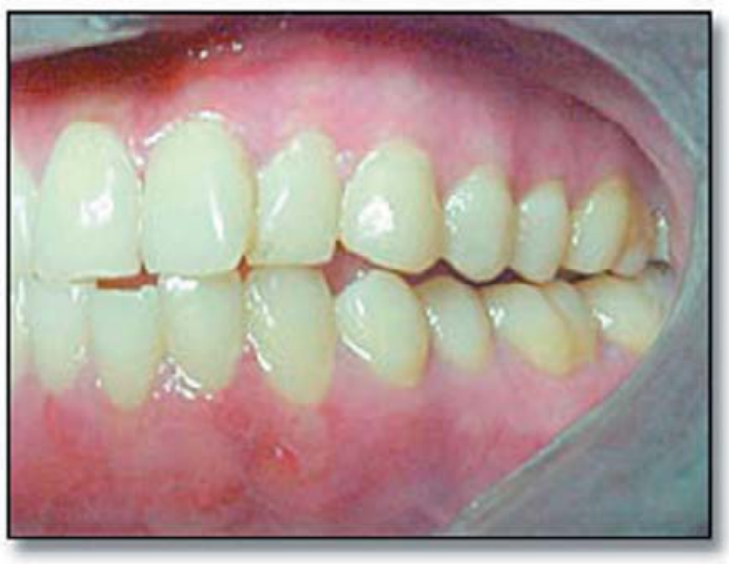

Figure 7. After 10 weeks. There is complete reduction in desquamation in all the sites except in the left canine region.

\section{Discussion}

Acrylic labial veneers were first advocated by Emslie $^{7}$ to improve the aesthetics in chronic periodontitis patients. L'Estrange and Strahan ${ }^{8}$ used them to carry periodontal dressings.

Wray and $\mathrm{McCord}^{3}$ described the use of acrylic labial veneers as a vehicle for delivering medication and as a way to improve the esthetics. They treated five female patients with clinically diagnosed desquamative gingivitis and stated acrylic labial veneers are useful adjuncts in the management of patients with desquamative gingivitis as they improve the aesthetics and protect the fragile gingival epithelium from further trauma. In their study they found no patient had become free of desquamative gingivitis by wearing this appliance, but it reduced the amount of erythema and desquamation to a considerable extent. 
Lilly et al. ${ }^{9}$ used mouth guard trays extending on to the gingiva as an alternative to restarting systemic prednisalone therapy. They were constructed for local delivery of Clobetasol $0.05 \%$ ointment placed in the mouth for 30 minutes twice daily.

Nisengard ${ }^{10}$ stated the vehicle for the topical steroid plays a role in its therapeutic effectiveness and patient acceptability. He noted occlusive therapy, the covering of the topical steroid with an impermeable wrap or plastic stints, improves the drug absorption and clinical efficacy.

Lorenzana et al. ${ }^{11}$ stated practitioners who are considering the use of plastic stents or any other appliance as a vehicle for corticosteroid therapy must keep in mind the fact that the constant flushing of the oral cavity by the saliva as well as the movement of the surrounding structures like the lips, tongue, and the buccal mucosa may affect the efficacy of the medicaments. They also stated the use of stents or any other appliance is contraindicated for up to 6 weeks if any surgery like connective tissue grafting has been performed to prevent pressure and necrosis on the graft.

Both systemic and topical corticosteroids have been used in the treatment of desquamative gingivitis. Systemic steroids are usually the mode of choice over topical application where there is systemic involvement. ${ }^{12}$ However, the turbulent oral environment and the difficulty in ensuring an adequate and long-term contact of the medicament to the affected sites usually complicate the use of topical therapy. ${ }^{3,13}$

Topical corticosteroids are often recommended to: (1) reduce the risk of local and systemic side effects; (2) avoid sudden discontinuation of steroids (in the systemic form), and (3) treat children, large areas of the body, or areas susceptible to steroid damage such as the face. ${ }^{10}$ They have been divided into 4 classes with class I being the least potent and class IV being the most potent or into 7 groups with group I being the most potent and group VII the least potent. Betamethasone dipropropionate and Clobetasol propionate are examples of very potent class IV topical steroids and group I topical steroids depending on the classification. Fluocinonide acetonide is an example of either a moderately potent class II $(0.01 \%)$ or a potent class III $(0.025 \%)$ drug by one of the classifications or group II (in $0.2 \%$ cream form, $0.5 \%$ cream, ointment or gel form) or group III (medication $(0.25 \%$ ointment) by the other classification. Triamcinolone acetonide is an example of a Group III drug. Group I and II steroids are generally applied b.i.d., while Group III to VII are often applied t.i.d or q.i.d. Generally, the more potent groups (I, II, and III) or classes (II, III, and IV) are currently used for treatment of the diseases. ${ }^{10}$

Many topical corticosteroids have been advocated in the treatment of desquamative gingivitis. Fluocinonide $0.025 \%-0.05 \%$, Clobetasol propionate $0.05 \%$ (2-3 applications for 9-24 weeks), or Triamcinolone acetonide $(0.1 \%-0.5 \%)$ aqueous rinses or creams are some of the treatment modalities, though argument exists regarding their sufficiency in the treatment of desquamative gingivitis. ${ }^{1,12,13}$ Topical therapy in itself is known to be sufficient. ${ }^{9,10,12}$

Corticosteroids are only minimally absorbed following application to normal skin or mucosa. Long-term occlusion, i.e., the use of impermeable films (like veneers) over skin or mucous membranes is an effective method of enhancing penetration yielding a ten-fold increase in absorption. ${ }^{14}$ The penetration of corticosteroids also increases when applied to inflamed skin or in severe exfoliative diseases. ${ }^{14}$ Corticosteroids with veneers were used in periods ranging from 2 weeks to 924 weeks. $^{3,12}$

The use of corticosteroids is, however, not without side effects and contraindications. The patient's present and past medical history must be taken into consideration. The patient is being constantly evaluated for signs of HypothalamicPituitary-Adrenal axis suppression ${ }^{13,15}$, Cushings syndrome ${ }^{13,15}$, or other relevant signs such as candidiasis because of immunosuppression. ${ }^{14}$

The patient has been using this appliance for the past four months. Using this treatment modality, there was a reduction in the erythema and the desquamation except in an area near the left lower canine region. This is in agreement with the findings of Wray et al. ${ }^{3}$ who stated no patient has become free of desquamative gingivitis by wearing veneers but has demonstrated a reduction of erythema and desquamation. Recurrence 
of the lesions after the appliance was discontinued has been reported. ${ }^{13}$ The patient was comfortable with the appliance and was satisfied with the esthetics. He is on recall visits with special emphasis on oral hygiene maintenance and is being regularly monitored for corticosteroid side effects.

\section{Summary}

We have described a case of desquamative gingivitis in a 24-year old male who was treated by the application of topical corticosteroid on the affect- ed surfaces which was then covered with a labial veneer in order to improve its absorption and efficacy. We suggest that acrylic labial veneer is a useful adjunct in the management of patients with desquamative gingivitis. It not only provides a suitable vehicle for the delivery of corticosteroids but also improves the aesthetics and aids in the protection of the fragile gingival tissues. However, a four-month follow up is a relatively short period to comment on the efficacy of labial veneers and further follow-up may be required.

\section{References}

1. Aguire A, Neiders MF, Nisengard RJ. Desquamative Gingivitis. In: Carranza FA, Newman MG, Takei HH. Carranza's Clinical Periodontology. W. B. Saunders Co., Philadelphia, 2001.

2. Meritt AH. Chronic Desquamative Gingivitis. J Periodontol 1933; 4: 30-34.

3. Wray D, McCord JF. Labial veneers in the management of desquamative gingivitis. Oral Surg Oral Med Oral Pathol. 1987 Jul;64(1):41-2.

4. Nisengard RJ, Neiders M. Desquamative lesions of the gingiva. J Periodontol. 1981 Sep;52(9):500-10.

5. Nisengard RJ, Rogers RS 3rd. The treatment of desquamative gingival lesions. J Periodontol. 1987 Mar;58(3):167-72.

6. Mobini N, Nagarwalla N, Ahmed AR. Oral pemphigoid. Subset of cicatricial pemphigoid? Oral Surg Oral Med Oral Pathol Oral Radiol Endod. 1998 Jan;85(1):37-43.

7. Emslie RD. A case of advanced periodontitis complex. Dent Practitioner. 1955;5: 432-433.

8. L'Estrange PR, Strahan JD. The wearing of acrylic periodontal veneers. Br Dent J. 1970 Feb 17;128(4): 193-4. No abstract available.

9. Lilly JP, Spivey JD, Fotos PG. Benign mucous membrane pemphigoid with advanced periodontal involvement: diagnosis and therapy. J Periodontol. 1995 Aug;66(8):737-41. Review.

10. Nisengard RJ. Periodontal implications: mucocutaneous disorders. Ann Periodontol. 1996 Nov;1(1): 401-38. Review. No abstract available.

11. Lorenzana ER, Rees TD, Hallmon WW. Esthetic management of multiple recession defects in a patient with cicatricial pemphigoid. J Periodontol. 2001 Feb;72(2):230-7.

12. Schully C, Carrozo M, Gandolfo S, et. al. Update on mucous membrane pemphigoid: a heterogeneous immune-mediated subepithelial blistering entity. Oral Surg Oral Med Oral Pathol Oral Radiol Endod. $1999 \mathrm{Jul} ; 88(1): 56-68$. Review.

13. Aufdemorte TB, De Villez RL, Parel SM. Modified topical steroid therapy for the treatment of oral mucous membrane pemphigoid.

Oral Surg Oral Med Oral Pathol. 1985 Mar;59(3):256-60.

14. Robertson DB. Mailbach HI. Dermatologic Pharmacology. In: Katzing BG. Basic and Clinical Pharmacology. McGraw Hill., New York, 2004.

15. Vincent SD. Lilly GE. Baker KA. Clinical, historic, and therapeutic features of cicatricial pemphigoid. A literature review and open therapeutic trial with corticosteroids. Oral Surg Oral Med Oral Pathol. 1993 Oct;76(4):453-9. Review. 
About the Authors

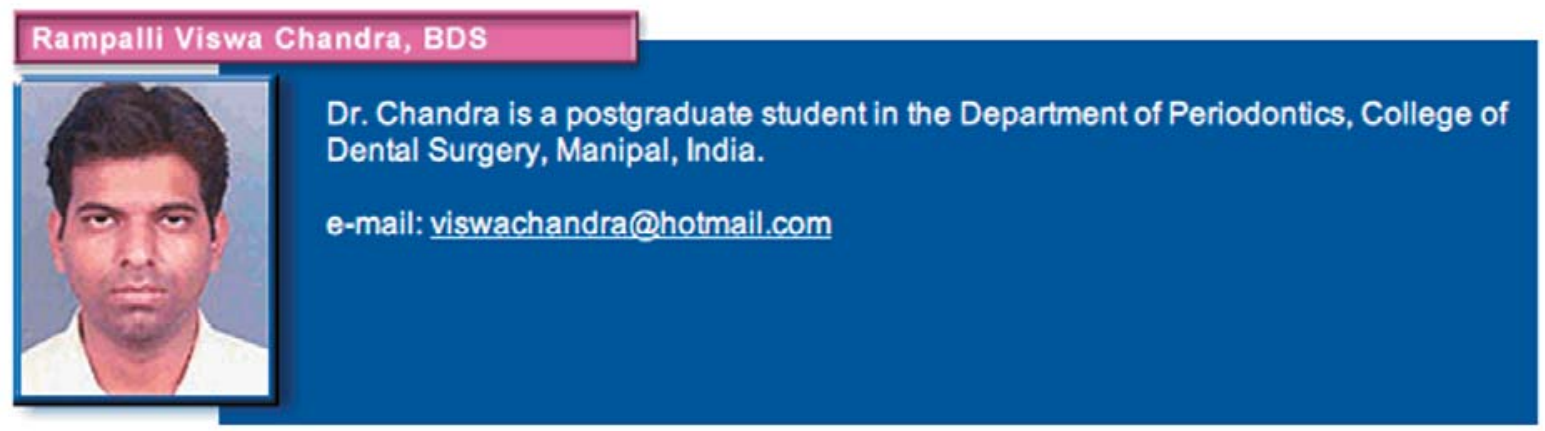

\section{Pratibha Pandurang, MDS}

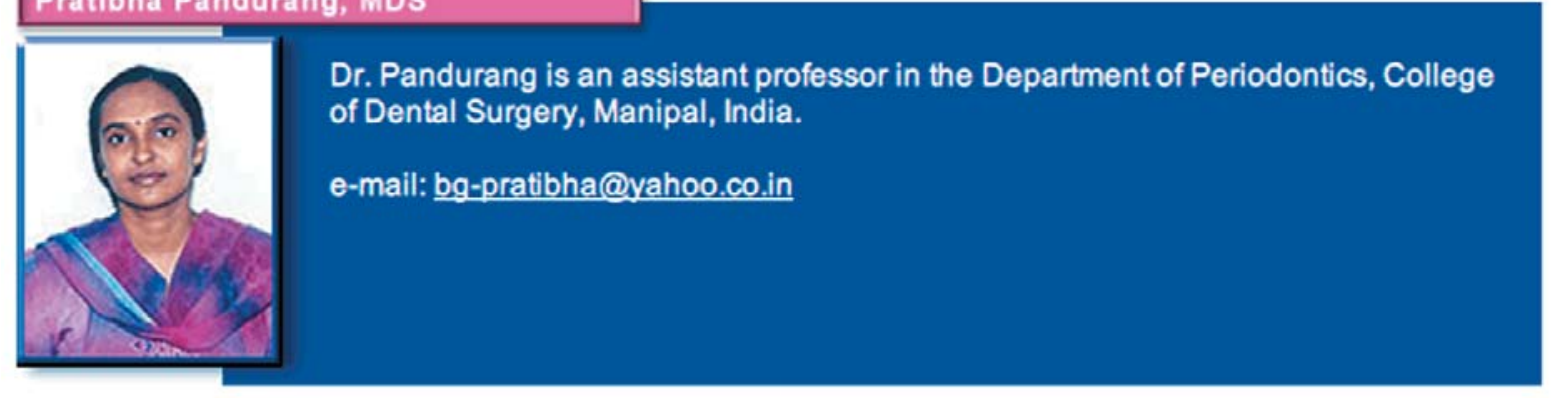

\section{Khandige Mahalinga Bhat, BSc, MDS}

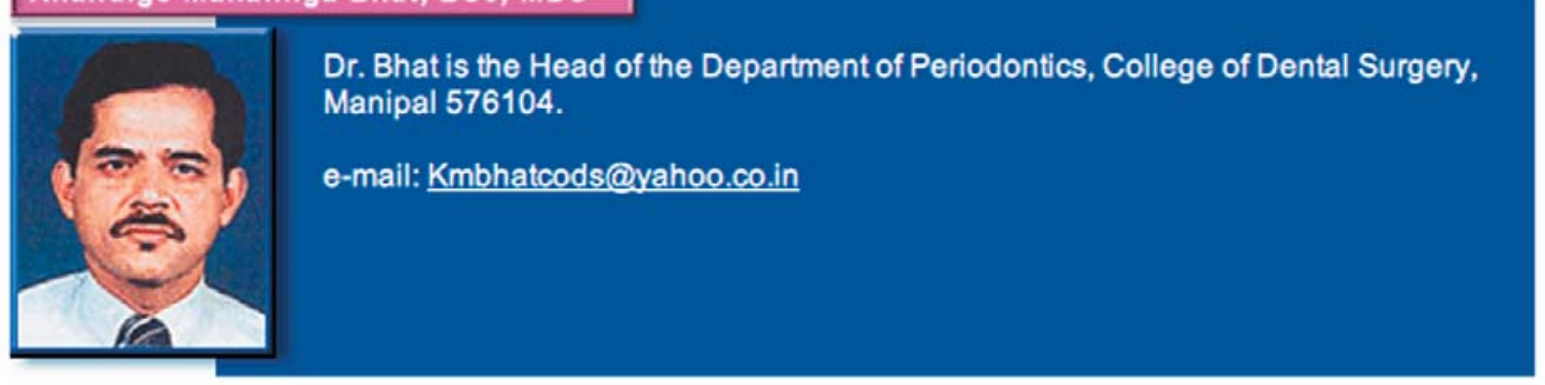

